\title{
Contacts and lines SEM image metrology with SMILE
}

\author{
Iacopo Mochi, Michaela Vockenhuber, Timothée Allenet, and Yasin Ekinci \\ Paul Scherrer Institut, Villigen, Switzerland
}

\begin{abstract}
SMILE is a free and open-source software for the analysis of SEM images of lines and spaces patterns. We developed SMILE specifically to provide reliable metrology for images of wafers obtained with EUV interference lithography in the framework of the EUV resist screening program at the Paul Scherrer Institute (PSI). In its original version, SMILE offered the possibility to measure the critical dimension of the lines and their unbiased line-width roughness. Since its release, the software user interface and core functionalities have been substantially upgraded. In particular, SMILE is now capable of reporting unbiased line-edge roughness, performing primary statistical analyses of metrics evaluated over multiple images, and analyzing SEM images of contact arrays. In this paper, we will discuss the new functionalities of SMILE and the algorithms used to detect the contacts' edge profile.
\end{abstract}

Keywords: SEM, resist, metrology, LWR, LER, critical dimension, contacts, edge detection.

\section{INTRODUCTION}

SMILE (SEM-Measured Image Lines Estimator) is a free software for the analysis of scanning electron microscopy (SEM) images. ${ }^{1}$ It was originally designed to support the EUV resist screening program run at the Paul Scherrer Institute (PSI). ${ }^{2}$ This program relies on the XIL-II beamline facility at the Swiss Light Source, the PSI synchrotron. The beamline is equipped with an interference lithography end station that users can access to print periodic nano-structures like contact arrays or line gratings on wafers coated with EUV photoresist. ${ }^{3}$ To gather information about the resist quality, the wafers are inspected with a SEM and relevant metrology information is gathered using the SMILE software. The software features different edge detection algorithms for lines and contacts which can be customized and extended to address user-specific needs.

Since SMILE's first public release, ${ }^{1}$ we relied on users feedback to improve its stability, and to upgrade it with different features. Here we present some of the new features of SMILE 2.0, the latest software version which is the result of this upgrade process.

SMILE is developed in MATLAB ${ }^{\circledR}$ and it is structured as a main program calling a series of script and functions. While in the first version, the main program is a function, SMILE 2.0 is designed as MATLAB application. This made it possible to quickly redesign the user interface to improve the user experience and to include access to new functionalities.

\section{SMILE 2.0 FEATURES AND UPGRADES}

The core functionalities of SMILE, including the possibility to choose from three different edge detection functions, the image gradient and rotation correction and the lines distortion correction, have remained unchanged. However, we added the support for single line or trench analysis, to enable the evaluation of LER and LWR on images of large structures or obtained with high magnification. The user workflow is the same as in the previous version and consist of three simple steps: data selection, parameters setting and image processing.

Further author information: E-mail: iacopo.mochi@psi.ch 


\subsection{SMILE workflow and GUI}

The graphical user interface (GUI) has been redesigned to include access to the new software features and to improve the user experience. As in the previous version, the GUI starting screen is the Images tab shown in figure 1. The first step in the image analysis workflow is the selection of the folder containing the images to be processed. This is done pressing the Images Folder button at the top of the Images tab. The software will load in memory all the files with TIF or JPG extension found in the specified folder and will populate the first three columns in the two tables on the left of the screen. We trained a convolutional neural network with hundreds of images of structures obtained with our interference lithography platform, to recognize lines and contact arrays. The software uses this network to categorize the images automatically and lists the images with lines and contacts array in the top and bottom table respectively. The user can always toggle the image type in case of error. Once the images are loaded in memory, the user can scroll the items in the two tables and visualize the images in the top left figure.

The next step is the parameters' selection. This is done in the Parameters tab shown in figure 2. In the redesigned GUI, the parameters are grouped in different boxes according to their function. Among the image pre-processing parameters we added a manual rotation adjustment angle, to correct the alignment of images with lines array that are visibly not vertical. Once the rotation angle error is lower than about 5 degrees, if the auto rotation alignment box is selected, the software will perform the final angle adjustment. A group of contact-specific parameters has been added to control the edge detection procedure for contact array images. These new parameters will be discussed in detail in section 2.4. When a suitable set of parameters has been determined, the user can save it as a MATLAB binary file and retrieve it for future use with the Save parameters and Load parameters buttons.

The final step of the SMILE workflow is the image processing, which can be started from the Images tab by pressing the Process images button. The user can select a subset of the available images by toggling the tick boxes in the first column of the data tables. The software will analyze the selected images according to their type and to the current parameter set. As the analysis is carried out, the tables are filled with the measured data. The data processing can be paused, resumed or stopped using the control buttons that will appear below the progress gauge at the top of the window. The lines or contact contours are plotted on top of the processed image and displayed on the top left figure. When the analysis is complete, the user can scroll the tables and click on any row. If the selected row contains an image that has been analyzed, the software displays the contour overlay plot on the top left figure. In case the image is a line array, the bottom left figure will display a plot of an user-selected metric. The available choices include the plot of the average CD of each line in the image array, the average height-difference correlation function and the power spectral density for the line width, or for the line edges. If the selected image is a contact array, the software will show a selection panel for contact-specific metrics including the contacts radius, its ellipticity, the angle of the best fitting ellipse and the standard deviation error of the difference between the profile and the fitted ellipse. The values of the metrics are represented by color patches superimposed to the contacts as shown in figure 7 .

\subsection{Edge-specific line edge roughness}

SEM setting and sample geometry can introduce a bias between the leading edge and the trailing edge of the lines. Systematic scanning anomalies can also be evidenced by the correlation of the two edges. Because of this, in some cases, it could be useful to analyze the right and left edge of the lines separately. ${ }^{4}$ For this reason, we introduced the possibility to measure the LER and the average correlation length for the leading edges and the trailing edges separately. It is also possible to display the LER PSD for trailing and leading edges in the bottom left figure in the Images tab window.

\subsection{Height difference correlation function model and fit}

In SMILE 2.0, the LWR and LER correlation lengths are estimated by fitting a Gaussian model ${ }^{5}$ to the measured height difference correlation function (HDCF). The HDCF model $g(x)$ is given by:

$$
g(x)=2 a\left(1-\exp \left(-(x / b)^{2 c}\right)\right)+d
$$




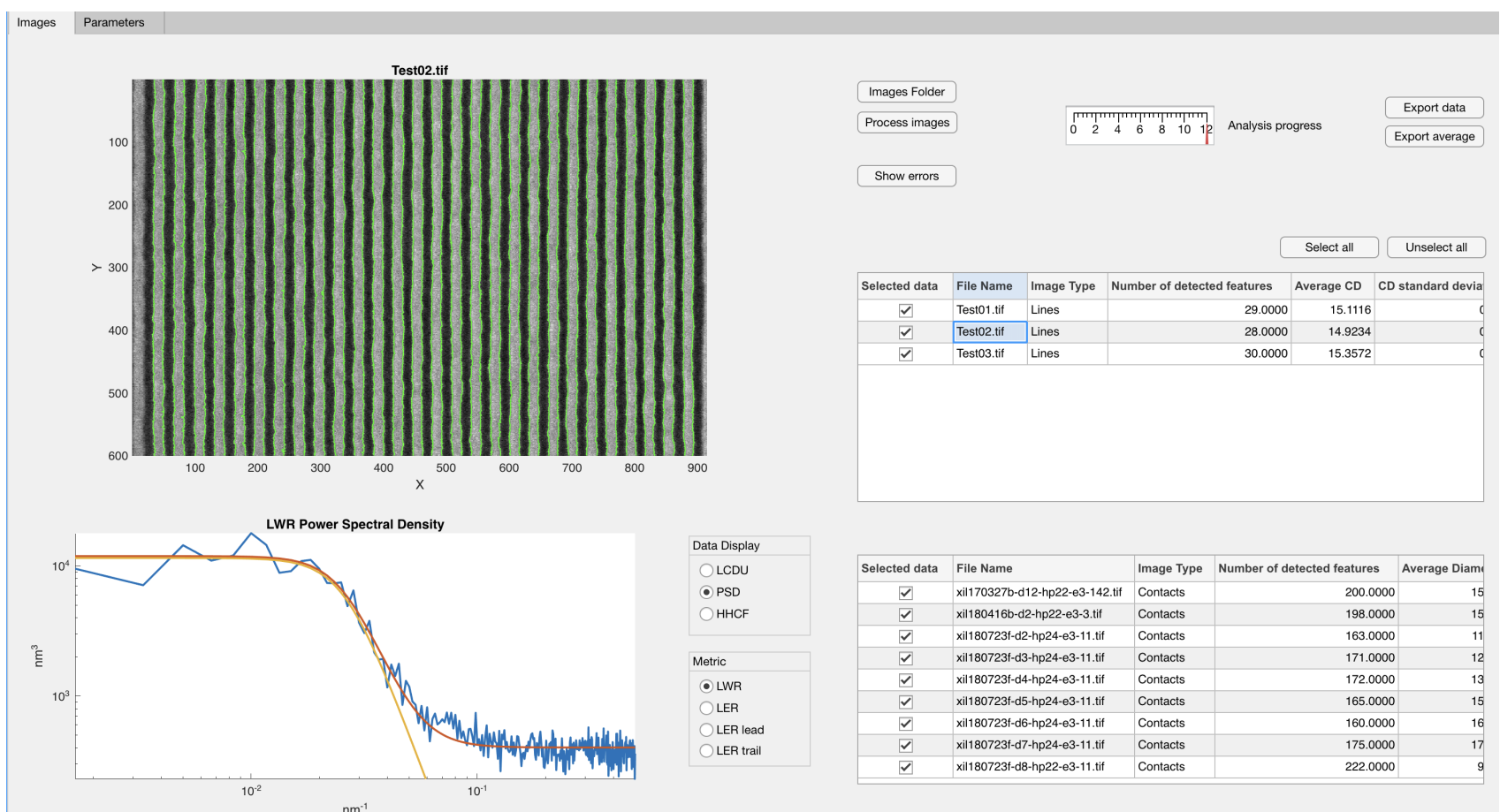

Figure 1. This is the Images tab screen of SMILE. From here, the user can select the images to measure, run the analysis and review and export the results.

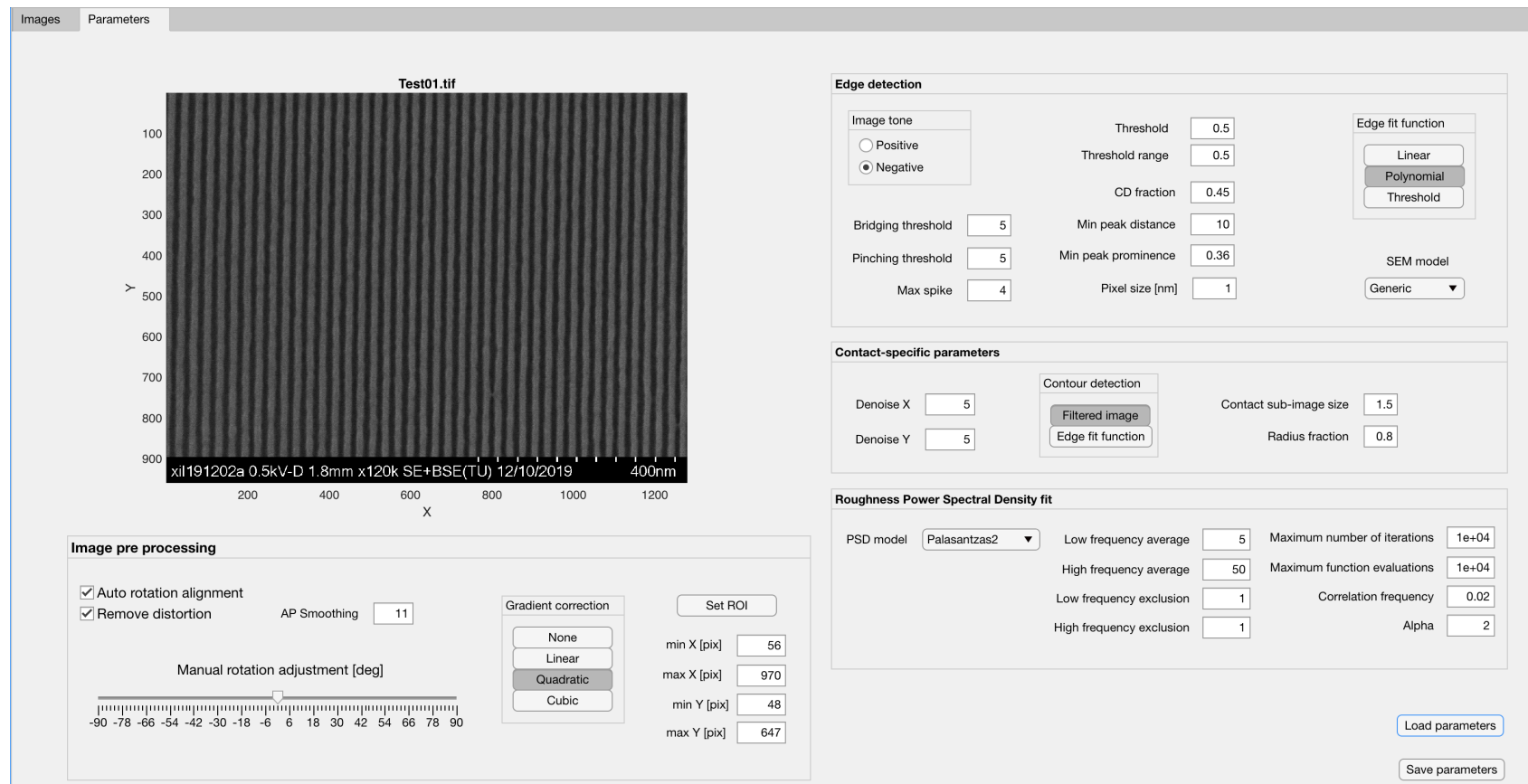

Figure 2. On the Parameters tab screen, the user can control the image pre-processing procedure, the edge detection algorithm behavior and the roughness analysis parameters. It is also possible to save and import a set of parameters for future use. 


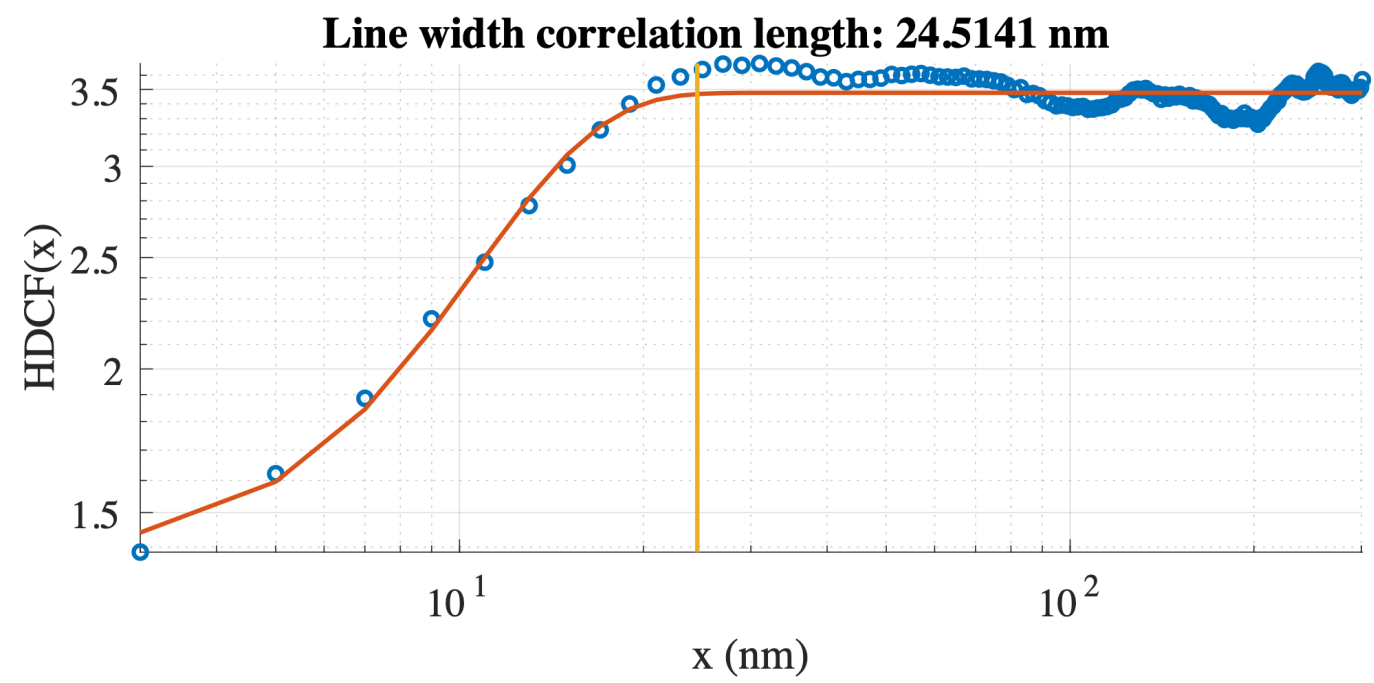

Figure 3. Height-difference correlation function fit (continuous curve) and correlation length estimate (vertical line).

where $x$ is the axial distance between two points in the profile, $a$ is the profile's standard deviation, $b$ is the correlation length, $c$ is the Hurst exponent and $d$ is the the biasing term due to the uncorrelated image noise. ${ }^{6}$ The HDCF, its fitted model and the estimated correlation length can be displayed on the bottom left picture of the Images tab (see figure 3) and are calculated for the line width, the line edges and the leading and trailing edges separately.

\subsection{Contact edge detection algorithms}

Contact array metrology is a new feature is SMILE 2.0. As for line and spaces arrays, the accurate measurement of the contacts edges is the a key factor for reliable metrology. The contact edge detection algorithm we adopted is a two-step process. The first step consists in estimating the general edge location. This is done by applying a Gaussian noise reduction filter to the image and measuring the contacts contour at the specified threshold as shown in figure 4. The strength of the filter along the $\mathrm{x}$ and $\mathrm{y}$ direction can be controlled by the user through the Denoise $X$ and Denoise $Y$ values in Contact-specific parameter group (see figure 2). This procedure yields the approximate contour, the average radius and the centroid location for each of the detected contacts. The profiles detected with this step can already be considered sufficiently accurate for some applications, however, in general is desirable to avoid any image filtering that could bias the results. For this reason, the user can instruct the software to perform an additional edge detection step by toggling the Edge fit function button in the Contour detection parameter box. In this case the software will use the edge fit function procedure selected in the Edge fit function box on the top right of the Parameters tab. The available options include a basic threshold approach, a linear fit and a polynomial fit. Each of the contacts identified in the previous step will be analyzed separately. SMILE extracts a sub image centered on the estimated contact centroid with a size specified in the Contact sub-image size field in radius units. For each point $\vec{p}$ of the profile estimated in the previous step, the program extract an image intensity profile along a line from the contact centroid $\vec{c}$ to $\vec{p}$ as shown in figure 5A. The profile is centered on $\vec{p}$ and has a length specified by the Radius fraction parameter in radius units. The profile is extracted from the contact image with a linear interpolation. The position of the edge $\overrightarrow{p^{\prime}}$ is estimated using the method selected in the Edge fit function box. In case Threshold is selected, the software performs a linear interpolation of the profile and selects the its intercept with the threshold value which is closest to $\vec{p}$. If Linear is selected (figure $5 \mathrm{~B}$ ), the a straight line is fitted to the edge profile and $\overrightarrow{p^{\prime}}$ is calculated as the intercept with the threshold value. If Polynomial is selected (figure 5C), the profile is fitted with a polynomial of 4 degree and $\overrightarrow{p^{\prime}}$ is estimated as the polynomial's intercept with the threshold value which is closest to $\vec{p}$.

\subsection{Contact analysis}

Once the contact contour has been detected, the software estimates the best fitting ellipse by minimizing the difference between the contour's $\mathrm{x}$ and $\mathrm{y}$ coordinates and the coordinates of the 5-parameter ellipse model 
A

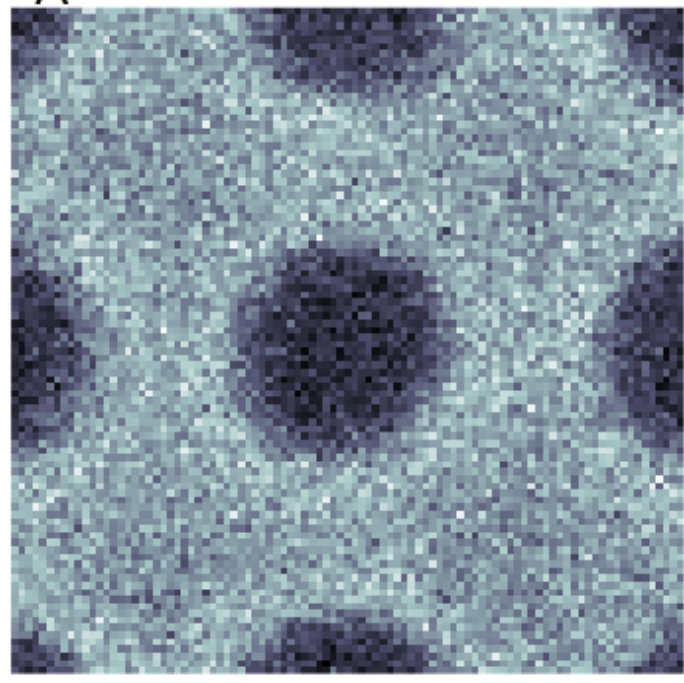

$20 \mathrm{~nm}$

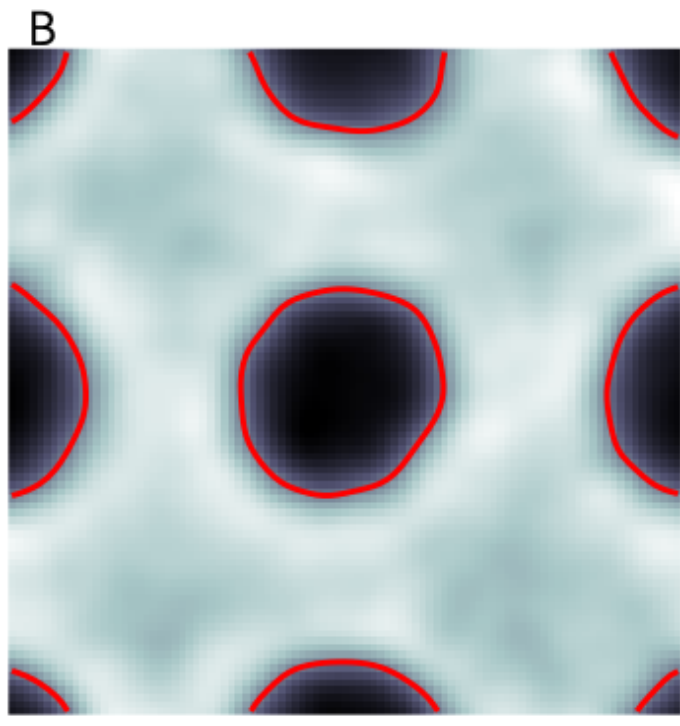

$20 \mathrm{~nm}$

Figure 4. A. Detail of a normalized contact array image. B. Noise filtered image and detected contact contours with a 0.5 threshold.

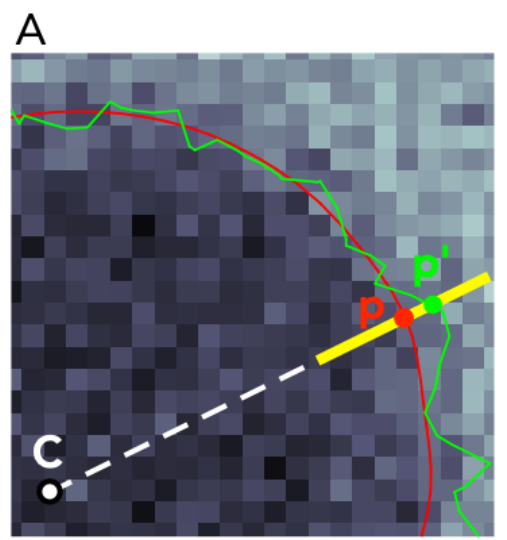

B

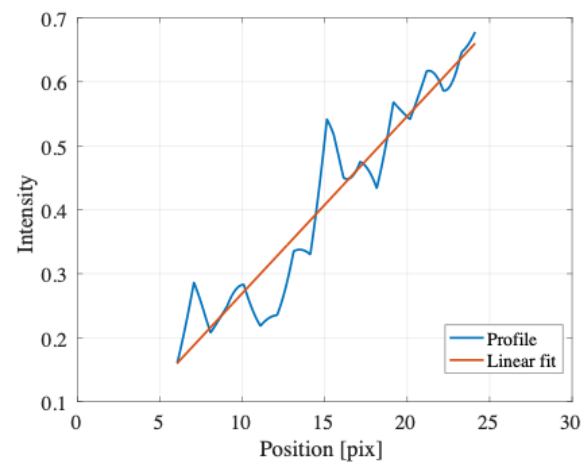

C

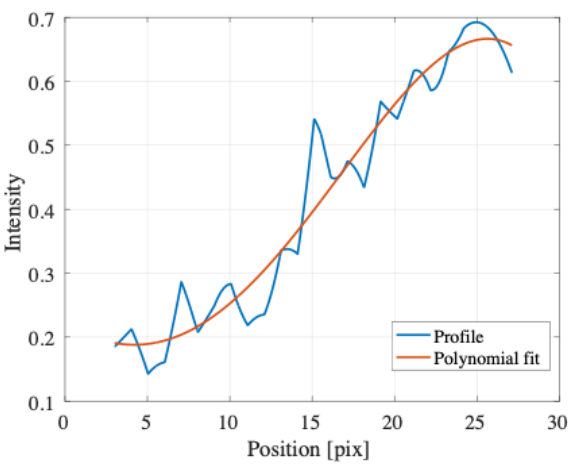

Figure 5. A. Contact edge detection procedure. For each point $p$ on the contour detected on the filtered image(red curve), the software extracts the image intensity over a radial segment originating from the image centroid $C$ and centered on $p$. B. A line is fitted to the radial intensity profile to calculate the intercept $p^{\prime}$ with the specified intensity threshold. C. A 4-degree polynomial is fitted to the radial intensity profile to calculate the intercept $p^{\prime}$ with the specified intensity threshold. 

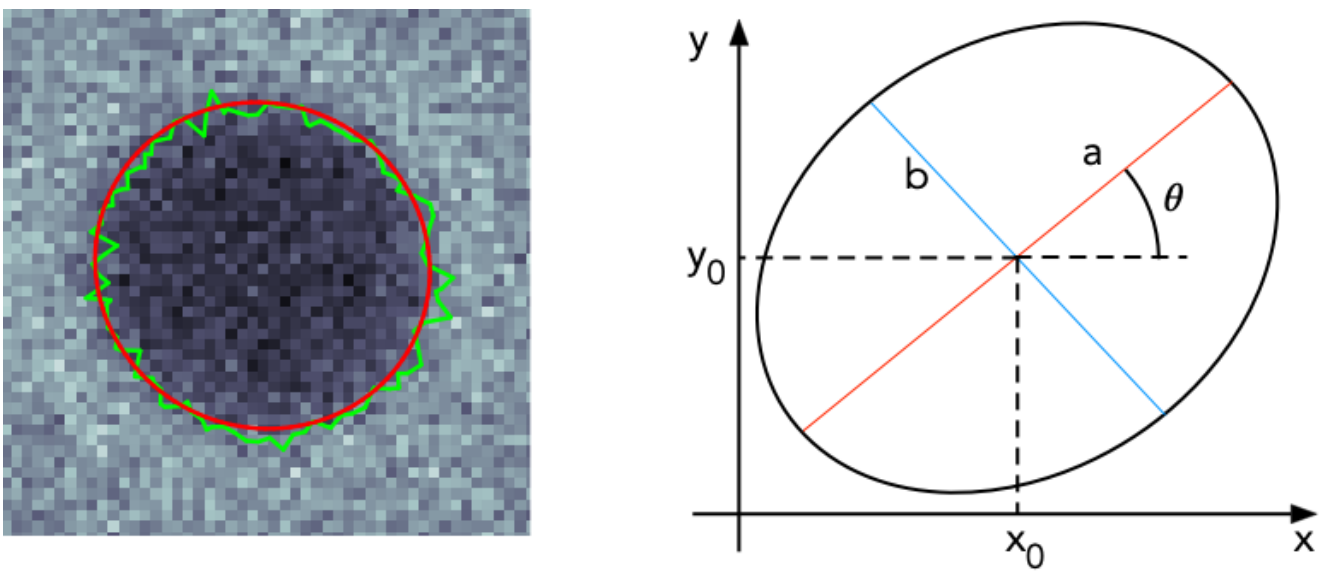

Figure 6 . The best fitting ellipse for the contact's profile is calculated using a model with 5 parameters: the major semiaxis $a$, the minor semiaxis $b$, the coordinates of the center $\left(x_{0}, y_{0}\right)$ and the angle thet $a$ between the ellipse's major axis and the $x$ axis

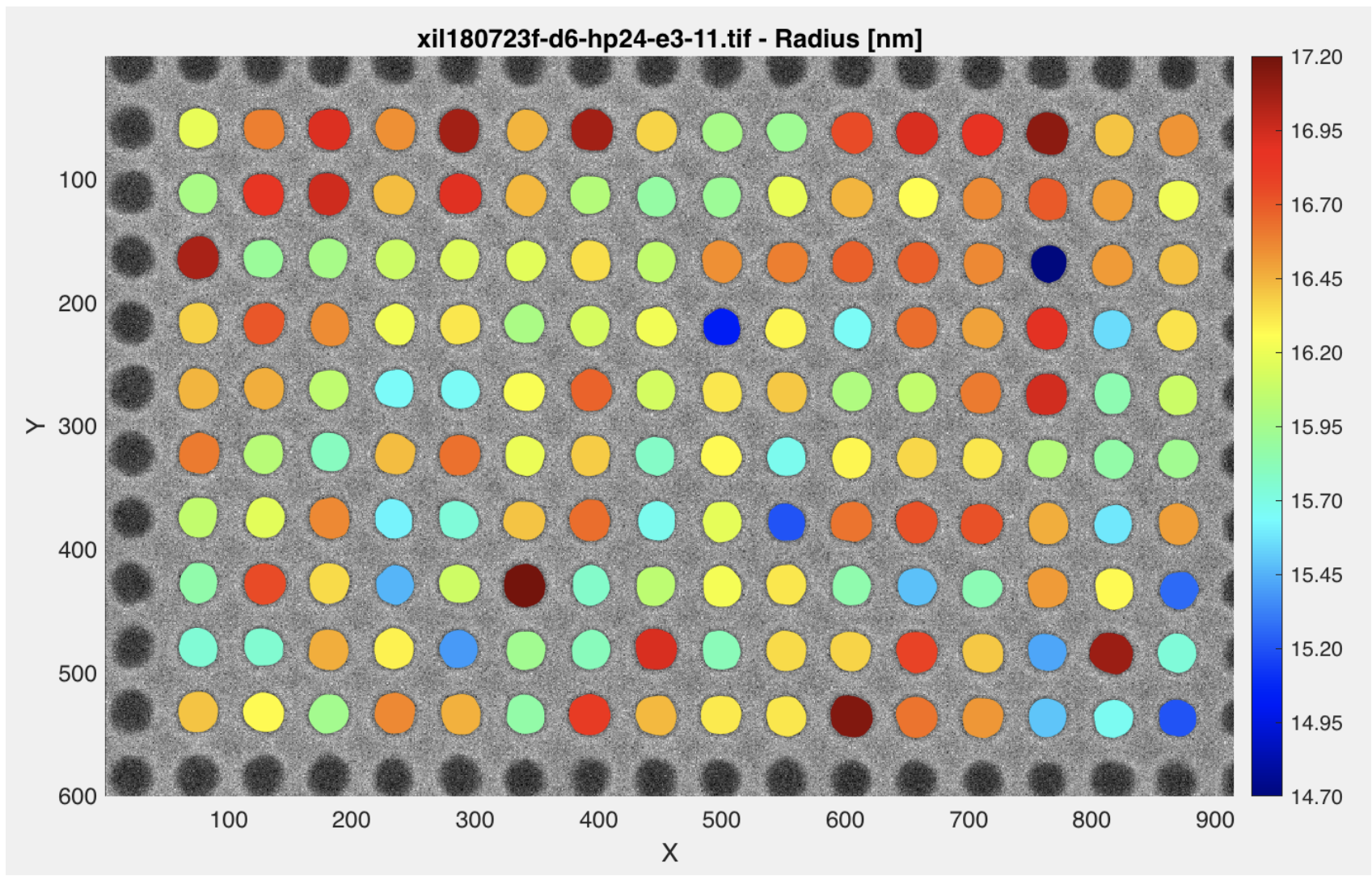

Figure 7. The average radius of each contact is visualized as a coloured patch to provide an immediate feedback on the presence of outliers in the array. The same visualization method is used to map the contacts' ellipticity, the angle of the fitted ellipse and the standard deviation of the difference between the contact profile and the fitted ellipse. 
described in equation 2:

$$
\vec{E}(\alpha)=\left[\begin{array}{cc}
\cos \theta & -\sin \theta \\
\sin \theta & \cos \theta
\end{array}\right]\left(\begin{array}{c}
a \cos \alpha+x_{0} \\
b \sin \alpha+y_{0}
\end{array}\right)
$$

where $a$ and $b$ are the two semi axes, $x_{0}$ and $y_{0}$ are the coordinates of the center, and $\theta$ is the rotation angle as shown in figure 6 . From the fitted model, the software extracts the ellipticity, the rotation angle and the standard deviation error of the contact profile. If $a$ and $b$ are the major and minor semi axis respectively, the ellipticity is defined as:

$$
e=\sqrt{\frac{a^{2}-b^{2}}{a^{2}}}
$$

and it can be used to map out field dependent aberrations in the image which may originate from the projection optics, from the photomask or, in the case of interference lithography where no optics or photomask are involved, from the SEM settings. The ellipse rotation angle provides information on the biasing direction for profiles with a significant ellipticity value. The standard deviation error is calculated as the standard deviation of the difference between the measured profile and fitted ellipse. It gives a measure of the irregularity, or roughness, of the contact profile. SMILE allows to visualize a map of these metrics overlaying coloured patches on the contact array image as shown in figure 7 .

\section{CONCLUSIONS}

We discussed the upgrades in the second version of our SEM image metrology software, SMILE 2.0. SMILE was developed for the analysis of SEM images of photoresists and wafer structures and it is the offical tool for the analysis of SEM image collected within the framework of the PSI EUV resist screening program. SMILE 2.0 is still under testing, but this new version includes several new features including a redesigned user interface, the ability to measure the LER of the leading and trailing edges of the lines separately, and to detect contact edges and perform basic metrology on contact array images.

SMILE 2.0 remains an an open source software and, as its previous version, is being developed using MATLAB (Release 2020b). However, we make it available to the public as source code and as an executable that can be installed and run without the need of a MATLAB license

SMILE 2.0 is still under testing, and, for the moment, it is possible to obtain the current version by contacting the authors. We are working on a web page were we will post the link to the new stable releases of the software binaries and source code.

We hope that with SMILE we can contribute to the lithography community by offering a open-source metrology software which can be improved and expanded by single users and by the community at large.

\section{ACKNOWLEDGMENTS}

The authors wish to thank all the users and early adopters of SMILE for their support and feedback.

\section{REFERENCES}

[1] Mochi, I., Vockenhuber, M., Allenet, T., and Ekinci, Y., "Open-source software for SEM metrology," in [Photomask Technology 2020], Preil, M. E., ed., 11518, 58 - 67, International Society for Optics and Photonics, SPIE (2020).

[2] Allenet, T., Wang, X., Vockenhuber, M., Yeh, C.-K., Mochi, I., Santaclara, J. G., Lent-Protasova, L. V., and Ekinci, Y., "Progress in EUV resist screening towards the deployment of high-NA lithography," in [Extreme Ultraviolet (EUV) Lithography XII], Felix, N. M. and Lio, A., eds., 11609, 34 - 43, International Society for Optics and Photonics, SPIE (2021).

[3] Mochi, I. and Ekinci, Y., "Lensless euv lithography and imaging," Synchrotron Radiation News 32(4), 22-27 (2019).

[4] Mack, C. A., Lorusso, G. F., and Delvaux, C., "Diagnosing and removing CD-SEM metrology artifacts," in [Metrology, Inspection, and Process Control for Semiconductor Manufacturing XXXV], Adan, O. and Robinson, J. C., eds., 11611, 300 - 318, International Society for Optics and Photonics, SPIE (2021). 
[5] Palasantzas, G., "Roughness spectrum and surface width of self-affine fractal surfaces via the k-correlation model," Phys. Rev. B 48, 14472-14478 (Nov 1993).

[6] Papavieros, G., Constantoudis, V., and Gogolides, E., "Allowable SEM noise for unbiased LER measurement," in [Metrology, Inspection, and Process Control for Microlithography XXXII], Ukraintsev, V. A., ed., 10585, 471 - 480, International Society for Optics and Photonics, SPIE (2018). 